\title{
Codebuch: Hate Speech im Internet 3/2019
}

\section{Annotationsbasierte Inhaltsanalyse von Nutzerkommentaren zum Thema Flucht und Migration aus März 2019}

im Rahmen des BMBF-Verbundprojekts „NOHATE - Bewältigung von Krisen öffentlicher Kommunikation im Themenfeld Flüchtlinge, Migration, Ausländer“ (01UG1735AX)

Sünje Paasch-Colberg, Christian Strippel, Laura Laugwitz, Martin Emmer \& Joachim Trebbe Institut für Publizistik- und Kommunikationswissenschaft, Freie Universität Berlin

\section{Inhaltsverzeichnis}

Kontext und Erkenntnisinteresse $\quad 2$

Untersuchungsmaterial und Analyseeinheit 2

Strukturierte Annotation mit BRAT (Manual) 3

Übersicht: Variablen und Ausprägungen $\quad 5$

$\begin{array}{ll}\text { Annotationsregeln } & 7\end{array}$

\section{Hinweis}

Dieses Dokument enthält potenziell beleidigende und verstörende, insbesondere rassistische und islamophobe Textpassagen. Sie dienen ausschließlich der Veranschaulichung der hier dokumentierten Codieranweisungen und spiegeln in keiner Weise die Meinung der Autor*innen wider.

\section{Zitation}

Paasch-Colberg, S., Strippel, C., Laugwitz, L., Emmer, M., \& Trebbe, J. (2021). Codebuch: Hate Speech im Internet 3/2019 - Annotationsbasierte Inhaltsanalyse von Nutzerkommentaren zum Thema Flucht und Migration aus März 2019 [Codebuch]. Freie Universität Berlin. 


\section{Kontext und Erkenntnisinteresse}

Hintergrund dieser Studie sind die aktuell viel diskutierten Probleme in öffentlichen Kommentarspalten und Diskussionsforen im Internet: War mit der Kommentarfunktionen auf Nachrichtenseiten, mit Blogs und sozialen Netzwerken die Hoffnung auf eine stärkere politische Partizipation und eine zunehmende Demokratisierung des öffentlichen Raumes verbunden, hat sich die Sicht auf solche Angebote stark gewandelt. In großem Umfang werden diese Diskussionsräume von hasserfüllten, beleidigenden Kommentaren gestört, die demokratische Werte wie die Akzeptanz abweichender Meinungen missachten. Ein aktuelles Beispiel dafür ist die Diskussion um die Themen Flucht und Migration, die die politische Öffentlichkeit derzeit intensiv beschäftigen. Befürchtet wird, dass sich hasserfüllte Kommentare negativ auf die Meinungsbildung und den gesellschaftlichen Zusammenhalt auswirken können, da sie darauf abzielen, bestimmte gesellschaftliche Gruppen von der öffentlichen Diskussion auszuschließen und zum Schweigen zu bringen. Zudem gibt es Hinweise darauf, dass solche Formen der Hassrede zum Aufstieg rechtsextremer Parteien und der Zunahme ausländerfeindlicher Straftaten beitragen.

Diese Studie ist Teil des vom BMBF geförderten Verbundprojekts „NOHATE - Bewältigung von Krisen öffentlicher Kommunikation im Themenfeld Flüchtlinge, Migration, Ausländer" (Förderkennzeichen: 01UG1735AX). Das Ziel dieses Projekts besteht darin, die Häufigkeit und Intensität von Hasskommentaren in diversen partizipativen Medienangeboten im Internet empirisch zu untersuchen und auf dieser Grundlage Hypothesen zu den Ursachen und Dynamiken von Hasskommentaren im Internet zu prüfen. Die in diesem Codebuch beschriebene annotationsbasierte Inhaltsanalyse von Nutzerkommentaren zu den Themen Flucht und Migration (Kommentaranalyse) schließt an eine qualitative Befragungsstudie mit Community Manager*innen in Deutschland und eine im August 2018 durchgeführte annotationsbasierte Inhaltsanalyse von Online-Diskussionen zum Thema Flucht und Migration an. Die in der Kommentaranalyse erhobenen Daten dienen als Ergänzung dieser ersten Inhaltsanalyse und als Trainingsdatensatz für die Entwicklung eines Algorithmus zur automatischen Erfassung und Klassifizierung von Hasskommentaren.

Die Kommentaranalyse verfolgt innerhalb des NOHATE-Projekts die folgenden Ziele:

1. Identifizierung von Hate-Speech-Aussagen innerhalb von Nutzerkommentaren.

2. Inhaltliche Dimensionierung von Hasskommentaren, z.B. anhand inhaltlicher, sprachlicher oder formaler Besonderheiten oder den von Hate Speech adressierten Personen(gruppen).

\section{Untersuchungsmaterial und Analyseeinheit}

Untersuchungsgegenstand der Kommentaranalyse sind öffentliche Nutzerkommentare zum Thema Flucht und Migration in Kommentarspalten auf journalistischen Webseiten und Social-Media-Angeboten, deren Fokus auf dem Austausch und der Diskussion von Inhalten zwischen Nutzer*innen liegt. Anhand der vier Auswahlkriterien (1) Reichweite, (2) Bedeutung des Angebots für die öffentliche Diskussion des Themas Flucht und Migration (gemessen anhand der Anzahl von Kommentaren zum Thema), (3) Varianz verschiedener Diskursarchitekturen sowie (4) Häufigkeit von Hate Speech in Kommentaren (basierend auf den Ergebnissen der Inhaltsanalyse im August 2018) wurden in einem mehrstufigen, bewussten Auswahlprozess folgende Angebote und Plattformen in die Stichprobe aufgenommen:

1. Nachrichtenseiten: Compact Online, Epoch Times, Focus Online

2. Facebook: Epoch Times, Focus Online, Welt, Zeit Online

3. YouTube: ARTEde, BILD, COMPACTTV, DW Deutsch, Epoch Times Deutsch, euronews (de), KenFM, Laut Gedacht, MrMarxismo, Oliver Flesch, RT Deutsch, tagesschau, Tagesschlau

4. Weblogs: PI-news.net 
Inhaltliches Aufgriffskriterium: Mithilfe einschlägiger Suchbegriffe wurden die Angebote in einem ersten Schritt im unten genannten Untersuchungszeitraum nach Beiträgen durchsucht, die inhaltlich Bezug auf das Thema Flucht und Migration nach Deutschland nehmen. In einem zweiten Schritt wurden für die so ausgewählten Postings und Beiträge alle dazugehörigen Kommentare erfasst. Auf diese Weise wird der Diskussionsverlauf zu einem thematisch relevanten Beitrag in seiner Gänze erfasst.

Untersuchungszeitraum: 01.-31.03.2019 (1 Monat)

Analyseeinheit: Im Rahmen der Kommentaranalyse erfolgt die Annotation auf der Ebene von Bewertungen: Innerhalb der Nutzerkommentare werden Bewertungen identifiziert und annotiert. Die Analyseeinheit dieser Kommentaranalyse ist demnach die einzelne Bewertung.

\section{Strukturierte Annotation mit BRAT (Manual)}

In dieser Studie wird mit der strukturierten Annotation als Variante der Textanalyse gearbeitet. Dabei werden Texte auf im Vorfeld festgelegte theoretische Konstrukte hin untersucht, allerdings geschieht dies nicht durch das Eintragen von Zahlencodes in eine Tabelle (wie bei der standardisierten Inhaltsanalyse), sondern durch das Markieren einzelner Textpassagen und der Zuweisung von Attributen zu diesen Textstellen. Dieses Verfahren ist der computergestützten, qualitativen Inhaltsanalyse (z.B. mit MAXQDA) sehr ähnlich. Allerdings werden die Attribute nicht induktiv vergeben, sondern sind bereits vorab definiert. Für dieses Verfahren wird in der vorliegenden Studie auf die Annotationssoftware BRAT (https://brat.nlplab.org/) zurückgegriffen.

BRAT ist eine browserbasierte Software mit visueller Nutzeroberfläche zur kollaborativen, strukturierten Annotation von Texten in allen Sprachen. Dazu muss keine gesonderte Software installiert werden. Es genügt ein Internetbrowser, bestenfalls Safari oder Chrome. Mobile Geräte mit berührungsempfindlichen Bildschirmen wie Smartphones oder Tablets werden nicht unterstützt. Das Programm liefert eine Vielzahl von Funktionen, die einfach und intuitiv eingesetzt werden können: Die Basisfunktion, die im Rahmen dieser Studie eingesetzt wird, ist die sogenannte "text span annotation“, also das Auswählen, Markieren und Labeln von Textstellen, das wie die Kommentarfunktion bei Microsoft Word funktioniert. Auf diese Weise können im Text so genannte „Entitäten“ definiert werden, die dann anhand vorgegebener Eigenschaften („,attributes“) weiter qualifiziert werden können.

Login

Rufen Sie in Ihrem Webbrowser (Safari oder Chrome) folgende URL auf: http://142.93.98.57/brat/. Im Falle des ersten Logins erscheint möglicherweise ein Fenster mit einem "Mini-Tutorial“, das Sie durch einen Klick auf das Kreuz rechts oben schließen können. Auch das folgende Fenster "Open“ können Sie schließen. Fahren Sie dann in dem noch geöffneten Fenster („No Document selected“) mit dem Cursor in die Ecke rechts oben. Es klappt automatisch ein Menü auf, in dem Sie rechts auf den Login-Button klicken. Dadurch öffnet sich ein Pop-up-Fenster, in das Sie Ihren Usernamen mit Passwort eingeben. Drücken Sie anschließend „OK“. Je nach Einstellung Ihres Browers könnte es sein, dass Sie sich fortan nicht noch einmal einloggen müssen. Haben Sie Ihren Usernamen oder das Passwort vergessen, kontaktieren Sie bitte die Studienleitung.

\section{Textdokument auswählen}

Durch das Drücken der Tabulatur-Taste $(\stackrel{\leftrightarrow}{\leftrightarrows})$ oder durch das Klicken auf den Collection-Button links oben in dem sich ausklappendem Menü gelangen Sie (zurück) zu dem Pop-up-Fenster "Open“, in dem Sie das zu annotierende Dokument auswählen können. Dazu klicken Sie einfach doppelt auf die entsprechenden Ordner $\square$ bzw. das entsprechende Dokument ( $\square$ ). In einem Ordner können Sie durch einen Klick auf die Zeile „.../“ in der Ordnerstruktur wieder in die nächsthöhere Ordnerebene wechseln. Bitte öffnen Sie nur die Ihnen zugeteilten Dokumente. 


\section{Annotieren}

In BRAT gibt es drei verschiedene Formen der Annotation: die Annotation von (1) Entitäten, (2) Relationen und (3) Ereignissen. Für die Annotation im Rahmen dieser Studie ist lediglich die Annotation von Entitäten relevant: Als Entität wird dabei die einzelne Bewertung definiert. Um sie in BRAT zu annotieren, wird der entsprechende Textabschnitt mit dem Cursor markiert („text span annotation“) und in dem daraufhin sich öffnenden Pop-up-Fenster („New Annotation“) im linken Bereich („Entity type“) der jeweilige Typ ausgewählt. Darüber hinaus können in dem Abschnitt „Entity attributes" die ausgewählten Entitäten noch mit Attributen weiter qualifiziert werden.

\section{Annotationen verlinken}

Für eine Erhebung mit mehreren Beteiligten ist es hilfreich, wenn man sich über die vorgenommenen Annotationen austauschen kann. Dazu bietet BRAT eine hilfreiche Funktion: Jede Annotation lässt sich über eine eigene URI verlinken und mit anderen teilen. Durch einen Doppelklick auf eine Annotation öffnet sich ein Fenster, in dem oben, rechts neben dem annotierten Text ein verknüpfter "Link" steht. Dahinter verbirgt sich die URI. Durch einen Rechtsklick auf diesen Link lässt sich durch die Auswahl des entsprechenden Menüpunktes der Link kopieren. Beim späteren Öffnen des Links wird die ausgewählte Annotation hervorgehoben, sodass sofort deutlich wird, um welche Annotation es sich handelt.

\section{Anmerkungen am Text vornehmen}

In BRAT besteht die Möglichkeit, offene Anmerkungen vorzunehmen, etwa wenn Probleme, Fragen oder Auffälligkeiten für die spätere Auswertung der Annotationen notiert werden sollen. Dazu wird eine entsprechende Textstelle markiert und die Anmerkung dann in dem sich öffnenden Fenster in das Freitextfeld "Notes" geschrieben. Annotiert wird die Anmerkung dabei als Entität, die jedoch nicht gesondert ausgewählt werden muss, da sie mit dem Öffnen des Fensters bereits markiert ist.

\section{Ablauf der Annotation}

Zunächst werden die Initialbeiträge gelesen und auf ihre thematische Relevanz geprüft. Im Anschluss daran werden die Kommentare von relevanten Beiträgen gelesen und nach Bewertungen gesucht. Jede identifizierte Bewertung wird annotiert und mithilfe der Attribute näher bestimmt. Der Ablauf der Annotation umfasst demnach die folgenden Schritte:

A. Vorbereitung

a. Öffnen von BRAT im Webbrowser: http://142.93.98.57/brat/ und Log-In

b. Bereitlegen des ausgedruckten Codebuchs

B. Initialbeitrag

a. Öffnen und Lesen des zu annotierenden Initialbeitrags in BRAT.

b. Vorprüfung auf thematische Relevanz

$\rightarrow$ wenn Beitrag thematisch relevant: weiter mit Punkt $\mathrm{C}$

$\rightarrow$ wenn Beitrag thematisch NICHT relevant, wird dies als Anmerkung vermerkt; die Kommentare zu diesem Beitrag werden übersprungen

C. Kommentar

a. Öffnen und Lesen des zu annotierenden Kommentars in BRAT.

b. Prüfung, ob der Kommentar zumindest eine Bewertung enthält.

c. Annotation der Bewertung(en) in BRAT: Markierung der Textstelle und Markierung der entsprechenden Attribute (BB1 - BB6e). 


\section{Übersicht: Variablen und Ausprägungen}

\begin{tabular}{|c|c|c|c|}
\hline Variable & Konstrukt & Ausprägungen & \\
\hline \multicolumn{4}{|c|}{ ENTITÄTEN } \\
\hline BB1 & Bewertungsrichtung & $\begin{array}{l}10 \text { Negative Bewertung } \\
20 \text { Ambivalente Bewertung } \\
30 \text { Positive Bewertung }\end{array}$ & ○ Auswahl \\
\hline \multicolumn{4}{|c|}{ ATTRIBUTE } \\
\hline BB2 & Bewertungsobjekt & $\begin{array}{l}00 \text { diffus/unbestimmt } \\
10 \text { Politischer Akteur: Deutschland } \\
21 \text { Politischer Akteur: Andere Länder } \\
22 \text { Politischer Akteur: International } \\
30 \text { Wirtschaftlicher Akteur } \\
41 \text { Akteure aus Kirche/Religion im Allg. } \\
42 \text { Islam im Speziellen } \\
50 \text { Soziale/Medizinische Akteure } \\
61 \text { Zivilgesellschaftlicher Akteur } \\
62 \text { Kriminelle/terroristische Akteure } \\
70 \text { Bildung/Forschung/Kultur } \\
80 \text { Akteur aus Medienbereich } \\
90 \text { Personen in Deutschland (o.M.) } \\
91 \text { Rechtsextreme in Deutschland } \\
92 \text { Personen mit Migrationshintergrund } \\
93 \text { Geflüchtete, Asylbewerber im Spez. } \\
95 \text { Personen aus dem Ausland }\end{array}$ & Dropdown \\
\hline BB3a & $\begin{array}{l}\text { Bewertungsdimension: } \\
\text { unspezifisch/übergreifend }\end{array}$ & $\begin{array}{l}0 \text { trifft nicht zu } \\
1 \text { trifft zu }\end{array}$ & Checkbox \\
\hline BB3b & $\begin{array}{l}\text { Bewertungsdimension: } \\
\text { Gesundheit }\end{array}$ & $\begin{array}{l}0 \text { trifft nicht zu } \\
1 \text { trifft zu }\end{array}$ & Checkbox \\
\hline BB3c & $\begin{array}{l}\text { Bewertungsdimension: } \\
\text { Intelligenz }\end{array}$ & $\begin{array}{l}0 \text { trifft nicht zu } \\
1 \text { trifft zu }\end{array}$ & Checkbox \\
\hline BB3d & $\begin{array}{l}\text { Bewertungsdimension: } \\
\text { Aussehen/Hygiene }\end{array}$ & $\begin{array}{l}0 \text { trifft nicht zu } \\
1 \text { trifft zu }\end{array}$ & Checkbox \\
\hline BB3e & $\begin{array}{l}\text { Bewertungsdimension: } \\
\text { Charakter/Verhalten }\end{array}$ & $\begin{array}{l}0 \text { trifft nicht zu } \\
1 \text { trifft zu }\end{array}$ & Checkbox \\
\hline BB3f & $\begin{array}{l}\text { Bewertungsdimension: } \\
\text { Kultur }\end{array}$ & $\begin{array}{l}0 \text { trifft nicht zu } \\
1 \text { trifft zu }\end{array}$ & Checkbox \\
\hline BB3g & $\begin{array}{l}\text { Bewertungsdimension: } \\
\text { Sexualität }\end{array}$ & $\begin{array}{l}0 \text { trifft nicht zu } \\
1 \text { trifft zu }\end{array}$ & Checkbox \\
\hline BB3h & $\begin{array}{l}\text { Bewertungsdimension: } \\
\text { Gesetzestreue }\end{array}$ & $\begin{array}{l}0 \text { trifft nicht zu } \\
1 \text { trifft zu }\end{array}$ & Checkbox \\
\hline BB3i & $\begin{array}{l}\text { Bewertungsdimension: } \\
\text { Wirkung auf Deutschland }\end{array}$ & $\begin{array}{l}0 \text { trifft nicht zu } \\
1 \text { trifft zu }\end{array}$ & Checkbox \\
\hline
\end{tabular}




\begin{tabular}{|c|c|c|c|}
\hline BB4 & Implikation & $\begin{array}{l}00=\text { keine Implikation } \\
11=\text { Umgang ohne Gewalt } \\
12=\text { Gewalt } \\
13=\text { Tötung }\end{array}$ & Dropdown \\
\hline BB5a & $\begin{array}{l}\text { Gruppenbezug: } \\
\text { Ethnie }\end{array}$ & $\begin{array}{l}0 \text { trifft nicht zu } \\
1 \text { trifft zu }\end{array}$ & Checkbox \\
\hline BB5b & $\begin{array}{l}\text { Gruppenbezug: } \\
\text { Religion }\end{array}$ & $\begin{array}{l}0 \text { trifft nicht zu } \\
1 \text { trifft zu }\end{array}$ & Checkbox \\
\hline BB5c & $\begin{array}{l}\text { Gruppenbezug: } \\
\text { Geschlecht }\end{array}$ & $\begin{array}{l}0 \text { trifft nicht zu } \\
1 \text { trifft zu }\end{array}$ & Checkbox \\
\hline BB5d & $\begin{array}{l}\text { Gruppenbezug: } \\
\text { Beruf }\end{array}$ & $\begin{array}{l}0 \text { trifft nicht zu } \\
1 \text { trifft zu }\end{array}$ & Checkbox \\
\hline BB6a & $\begin{array}{l}\text { Sprachliche Form: } \\
\text { Beschimpfung }\end{array}$ & $\begin{array}{l}0 \text { trifft nicht zu } \\
1 \text { trifft zu }\end{array}$ & Checkbox \\
\hline BB6b & $\begin{array}{l}\text { Sprachliche Form: } \\
\text { Metapher/Vergleiche }\end{array}$ & $\begin{array}{l}0 \text { trifft nicht zu } \\
1 \text { trifft zu }\end{array}$ & Checkbox \\
\hline BB6c & $\begin{array}{l}\text { Sprachliche Form: } \\
\text { Formale Verfremdung }\end{array}$ & $\begin{array}{l}0 \text { trifft nicht zu } \\
1 \text { trifft zu }\end{array}$ & Checkbox \\
\hline BB6d & $\begin{array}{l}\text { Sprachliche Form: } \\
\text { Abwertende Umschreibung }\end{array}$ & $\begin{array}{l}0 \text { trifft nicht zu } \\
1 \text { trifft zu }\end{array}$ & Checkbox \\
\hline BB6e & $\begin{array}{l}\text { Sprachliche Form: } \\
\text { Entmenschlichung }\end{array}$ & $\begin{array}{l}0 \text { trifft nicht zu } \\
1 \text { trifft zu }\end{array}$ & Checkbox \\
\hline
\end{tabular}




\section{Annotationsregeln}

\section{Vorprüfung: Relevanter Beitrag?}

Kommentare werden nur dann annotiert, sofern der zugehörige Initialbeitrag einen substantiellen Bezug zum Thema Flüchtlinge in und Migration nach Deutschland aufweist (d.h. es wird in mehreren Sätzen inhaltlich erörtert). Dieses Zugriffskriterium erfüllen solche Beiträge, in denen die (auf Dauer angelegte) Einwanderung von Menschen aus anderen Ländern nach Deutschland (unabhängig von Migrationsmotiven) sowie damit verbundene politische und gesellschaftliche Ereignisse, Stellungnahmen, Diskussionen und Entscheidungen thematisiert werden. Konkret umfasst das Thema Ereignisse, Stellungnahmen, Diskussionen und Entscheidungen zu den folgenden Themen:

- Einwanderungsbewegungen/Flucht nach Deutschland

- Asylpolitik in Deutschland

- Leben von Geflüchteten/Einwanderern in Deutschland, Zusammenleben

- Umgang mit Geflüchteten in der deutschen Ankunftsgesellschaft und Diskussionen darüber

\section{Folgende Beiträge erfüllen das Zugriffskriterium:}

- Beiträge über Fluchtursachen/Migrationsmotive (z. B. Bürgerkrieg, Not und Armut) erfüllen das Zugriffskriterium nur, wenn explizit ein Deutschlandbezug hergestellt wird.

- Beiträge über Menschen auf der Flucht, die Tätigkeiten von Schleusern oder Helfenden, wenn explizit ein Bezug zu Deutschland hergestellt wird, wobei dieser Bezug durch Akteure oder thematisch hergestellt werden kann (z. B. wenn sich ein deutscher Politiker zu Schleusern äußert oder thematisiert wird, wie sich Maßnahmen gegen Schlepper auf die Zahlen der Einwanderer nach $D$ auswirken könnten).

- Beiträge über Migration nach Europa (allgemein) oder in andere europäische Länder erfüllen das Zugriffskriterium nur, wenn explizit ein Deutschlandbezug hergestellt wird.

- Beiträge zu nicht-christlichen Religionen (z. B. Islam, Muslime) oder Kulturen erfüllen das Zugriffskriterium nur, wenn diese Themen explizit im Zusammenhang mit aktuellen Migrationsbewegungen nach Deutschland thematisiert werden.

- Beiträge über Rassismus in Deutschland, wenn diese Themen explizit im Zusammenhang mit aktuellen Migrationsbewegungen nach D thematisiert werden.

\section{Folgende Beiträge erfüllen das Zugriffskriterium nicht:}

- Beiträge über Tourismus oder Binnenmigration (d.h. innerhalb von Deutschland)

- Beiträge über Migration nach Europa bzw. in andere europäische Länder oder über Fluchtursachen und Ereignisse auf der Flucht ohne Deutschlandbezug

- Beiträge über Rassismus oder über nicht-christliche Religionen oder Kulturen ohne Bezug zu den jüngsten Migrationsbewegungen

- Beiträge, in denen das Thema Flucht und Migration nicht substantiell, sondern nur am Rande (mit wenigen Worten) erwähnt oder nur als ein Thema unter vielen behandelt wird.

\section{O Anmerkung}

Für den Fall, dass das Zugriffskriterium nicht erfüllt ist, wird im Kopf des Initialbeitrags die Anmerkung annotiert: „Zugriffskriterium nicht erfüllt“. Die Kommentare, die zu einem solchen nicht-relevanten Beitrag gehören, werden übersprungen und nicht annotiert. 
Hintergrund: Für Bewertungen bzw. wertende Aussagen über andere Personen oder Gruppen wird sich im Rahmen dieser Studie interessiert, um so verschiedene Formen von Inzivilität bis hin zu Hasskommentaren identifizieren und klassifizieren sowie sie von positiven oder relativierenden Bewertungen unterscheiden zu können.

Definition: Eine Bewertung ist eine Aussage, die ein Werturteil oder eine Meinung über andere Personen oder Gruppen (= Bewertungsobjekt) enthält, indem ihnen bestimmte positive und/oder negative Eigenschaften und/oder Verhaltensweisen (= Dimension) zu- oder abgeschrieben werden.

\section{Identifikation:}

Annotiert werden ausschließlich wertende Aussagen über Personen oder Gruppen. Bewertungen von bestimmten Sachverhalten, Ereignissen, Gegenständen, Regeln sowie abstrakten Objekten wie „das System" oder „Deutschland“ etc. werden nicht annotiert.

Eine Bewertung lässt sich dabei an den folgenden konkreten Merkmalen erkennen:

- Zuschreibung bestimmter Adjektive („kriminelle Moslems“, „Araber sind gut“)

- Wertende Subjektivierung („Scheißkanake“, „Lügner“, ,Gutmenschen“)

- Verbiale Verallgemeinerungen („,sie schubsen, treten, vergewaltigen“)

- Vergleiche mit Tieren oder Dingen („wie die Karnickel Rammelnde“, „Abschaum“)

- Assoziation positiver oder negativer Konsequenzen oder Folgen

- Implizite Bewertungen liegen in den folgenden Fällen vor:

- Positive oder neutrale Aussagen, die mit der Grundannahme verbunden sind, denen zufolge die positive Eigenschaft für das bezeichnete Objekt untypisch ist.

Beispiel: „Meine Putzfrau ist echt gut, obwohl sie Türkin ist.“ ( $\rightarrow$ ambivalente Bewertung)

- Ausdruck der Hoffnung, dass niemandem durch das Objekt ein Schaden entstehe (wie z. B. eine Vergewaltigung)

Beispiel: „Da will man nur hoffen, dass sie nicht einmal unangenehm mit ausschließlich testosterongesteuerten Jungmannen - z. B. geflüchteten ,Alleinreisenden' aus der Dritten Welt, die uns massenhaft mit ihrer Lebensfreude und Herzlichkeit bereichern - unkommod ,konfrontiert' wird."

- Rhetorische Fragen, die kein echtes Informationsbedürfnis einfordern, sondern eine als Frage getarnte wertende Aussagen über ein Objekt vermitteln können.

Beispiele: „Lässt sie sich von denen etwa auch noch vergewaltigen?“; „glaubt ihr wirklich der kelch der Vergewaltigung und ermordeten geht an euch vorbei?"

- Ironische Begriffe oder Aussagen, die im wörtlichen Sinne positiv sind, aber durchscheinen lassen, dass eigentlich das Gegenteil gemeint ist.

Beispiel: „bereichern“

- Historische Bezüge, die einen bestimmten Umgang mit Menschen suggerieren, z.B. die Erwähnung von Duschen, Öfen, Gaskammern.

Beispiel: „Warum erst verhaftet, gleich unter die Dusche. Das kostet den Steuerzahler am wenigsten“ 


\section{Abgrenzung zu Aussagen der Zustimmung/Kritik:}

Aussagen, die pauschal Zustimmung oder Kritik ausdrücken, sich aber nicht auf eine konkrete Eigenschaft oder Verhaltensweise einer anderen Person beziehen, werden nicht als Bewertungen einer Person oder Grunde verstanden und NICHT annotiert.

- Beispiele: „Da hat er recht“; „Sehr gutes Statement!!!“

\section{Abgrenzung zu Beschreibungen ohne klare Bewertung:}

Beschreibungen von Situationen, die keine klare Bewertung enthalten und auch nicht negative und positive Aspekte gegeneinander abwiegen, werden ebenfalls nicht als Bewertungen annotiert.

- Beispiele: „Aber Deutschland nimmt immer weiter auf und signalisiert so, setzt euch in die Boote, wenn ihr erstmal auf den ,Rettungsschiffen' seid, nehmen wir euch auf......."

\section{Annotation in BRAT:}

Bei der Annotation von Bewertungen bitte folgende Regeln beachten:

- Eine neue Bewertung wird prinzipiell immer dann annotiert, wenn es einen Wechsel des Bewertungsobjekts oder der Bewertungsrichtung (negativ, ambivalent, positiv) gibt.

- Nur ganze Sätze annotieren! Nicht einzelne Worte oder Satzteile. Erstreckt sich eine Bewertung über mehrere Sätze, werden sie alle in die Annotation miteinbezogen. Annotiert wird immer vom Satzanfang zum Satzende (inkl. Satzzeichen).

- Sonderfall: Wird ein Wort oder Bindestrich-Wort oder eine Kombination aus einem Adjektiv und einem Substantiv verwendet, das als solches bereits eine Bewertung ausdrückt, ohne dass noch weitere Worte oder Satzteile diese Bewertung spezifizieren, wird nur dieses Wort als Bewertung annotiert. Dabei spielt es keine Rolle, ob der Satz, in dem das Wort vorkommt, selber auch als Bewertung codiert wird oder nicht. Beispiele: „Er wird in der Geschichte von Deutschland mehr in Erinnerung bleiben, als Mehrkill. Hat er doch schon vor der Bedrohung gewarnt, als diese kaum sichtbar war"; "Schaut euch die Nazi-Altparteien nur an wie sie sich selber huldigen und die neuen Fluchtsimulanten-lager eröffnen!!!!“

- Die Annotation einer Bewertung beginnt mit dem ersten Satz, der diese Bewertung enthält, und endet, sobald ein Satz folgt, der entweder keine Bewertung mehr aufweist.

○ Ausnahme: Kleinere Einschübe (1-2 Sätze) ohne Bewertungen, z.B. zur Einordnung, Erläuterung oder Kontextualisierung, die zwischen Sätzen mit derselben Bewertung stehen, werden mit annotiert. Zudem werden Sätze, die zwar vor oder nach der eigentlichen Bewertung stehen, diese aber nachvollziehbarer machen (z. B. weil aus innen das Bewertungsobjekt hervorgeht), als zugehörig zur Sinneinheit mit annotiert.

- Nicht kleinteilig annotieren! Versuchen, Sätze mit derselben Bewertung zu verbinden. Im Falle eines Wechsels von positiven und negativen Bewertungen desselben Objekts durch denselben Sprecher innerhalb eines Satzes oder einer Sinneinheit wird „20-Ambivalente Bewertung" annotiert.

- Einschübe: Einschübe mit Bewertungen anderer Personen oder Gruppen, werden zuerst mit in die Annotation der vorigen und nachfolgenden Bewertung einbezogen und dann noch einmal eigens annotiert.

- Differenzierung von Gruppen: Wenn innerhalb einer Gruppe zwei Sub-Gruppen differenziert und einander gegenübergestellt werden (es aber keinen Wechsel im Be- 
wertungsobjekt gibt), wobei die eine Sub-Gruppe positiv und die andere negativ bewertet wird, dann werden zwei Bewertungen annotiert (eine positive und eine negative) - es wird KEINE ambivalente Bewertung codiert.

Beispiel: „Man schaue doch nur in die Sozialen brennpunkte in Berlin, da kann das jeder Gleichmacherklugscheisser live Studieren!! Aber sie werden sich hüten - die ewigen Falschmünzer des Gutmenschentums! Viel lieber reagieren diese mit ,edler Entrüstung', gegen diejenigen, die auf die Realitäten verweisen, und sie ist natürlich auch ,gut' genug, nach der Bestrafung der Realisten zu verlangen - man solle doch die Realisten zum schweigen bringen! ( $\rightarrow$ positive Bewertung der , Realisten') Es sind ja eh nur Populisten - also Idioten die das laut aussprechen, was auf der Hand liegt! Ich sage: Es ist Zeit allen Realitätsverweigerern, Gleichmachern und Gutmenschen das dreckige Lügenmaul zu stopfen" ( $\rightarrow$ negative Bewertung der 'Gutmenschen')

- Auch Wiederholungen annotieren! Wird eine Bewertung an einer Stelle des Textes wiederholt, die jedoch zu weit entfernt vom ersten Auftreten im Text liegt, sodass eine gemeinsame Annotation nicht möglich ist, wird die Annotation einfach noch einmal vorgenommen.

Annotation einer Bewertung

\section{O Bewertungsrichtung}

Zunächst wird für jede identifizierte Bewertung festgehalten, ob es sich um eine negative, eine positive oder eine ambivalente bzw. differenzierende Bewertung handelt.

Eine negative Bewertung liegt vor, wenn einer Person oder einer Gruppe negative Eigenschaften zugeschrieben werden oder ihr aufgrund ihres Verhaltens Verstöße gegen sozial akzeptierte Normen zugeschrieben werden. Wenn also gesagt wird, dass jemand etwas tut, dass den geltenden Normen und Regeln widerspricht, einen Schaden verursacht oder Eigenschaften hat, die als nicht wünschenswert gelten. Eine negative Bewertung liegt kann auch durch die Negierung einer positiven Bewertung ausgedrückt werden.

Eine ambivalente oder differenzierte Bewertung liegt vor, wenn der Kommentierende positive und negative Bewertungen des Objektes gegeneinander abwiegt oder einen Schaden/Nutzen an Bedingungen knüpft.

Aber Achtung! Dies ist keine Restkategorie für Bewertungen, die schwer zu codieren sind, weil man sich z. B. nicht ganz sicher ist, ob sie ironisch gemeint sind oder nicht.

\section{Positive Bewertung}

Eine positive Bewertung liegt vor, wenn einem Objekt Eigenschaften oder Verhaltensweisen zugeschrieben werden, die den sozial akzeptierten Normen und Regeln entsprechen bzw. als wünschenswert gelten. Auch die Zuschreibung eines Nutzens, der durch das Objekt entstehe, gilt als eine positive Bewertung. Eine positive Bewertung kann auch durch die Negierung einer negativen Bewertung ausgedrückt werden.

Zu ironischen Aussagen: Diese werden so codiert wie sie gemeint sind - eine positiv formulierte Bewertung, die negativ gemeint ist, wird als negative Bewertung annotiert (und umgekehrt). 


\section{Bewertungsobjekt}

Im zweiten Schritt wird für jede identifizierte Bewertung diejenige Akteursgruppe bestimmt, aus die Person(en) stammt bzw. stammen, der bewertet wird/werden.

00

diffus/unbestimmt

Das Bewertungsobjekt ist nicht eindeutig.

\section{Politischer Akteur: Deutschland}

Alle Politiker*innen (Kanzlerin, Minister*innen, MdB, MdL, Bürgermeister*innen) und alle politischen Organisationen, Institutionen, Gremien bzw. deren Repräsentanten oder Sprecher*innen (z.B. Regierung, Parlamente, Fraktionen, Opposition, Ministerien, Ausschüsse) in Deutschland auf nationaler, regionaler und kommunaler Ebene. Dazu zählt auch die politische Verwaltung (Ämter, Behörden), Gerichte, Polizei, Militär, Küstenwache, Nachrichtenbzw. Geheimdienste und Parteien (inkl. Vorstand, Funktionäre, Mitglieder); auch „die Politik" oder "die Politiker" oder Synonyme wie "Berlin" oder "aus Regierungskreisen", wenn sich dies auf Politiker*innen oder politische Organisationen in Deutschland bezieht.

1 Politischer Akteur: Andere Länder

Politiker*innen, Repräsentanten und politische Organisationen anderer Länder als Deutschland; auch Rebellengruppen, Aufständige etc.

\section{Politischer Akteur: International}

Internationale und supranationale Institutionen (und deren Unterorganisationen) wie die Europäische Union (auch „Brüssel“ oder "Straßburg“), Europäischer Rat, Europäischer Gerichtshof für Menschenrechte, Internationaler Strafgerichtshof (,Den Haag“), Frontex, NATO, Vereinte Nationen (UNO), Flüchtlingshilfswerk (UNHCR)

\section{Wirtschaftlicher Akteur (national \& international)}

Nationale, ausländische und Internationale Unternehmen, Firmen, Banken, Organisationen, Interessensverbände und Gewerkschaften sowie deren Repräsentanten (Funktionäre, Sprecher*innen, Vorstände etc.)

1 Akteure aus Kirche/Religion im Allgemeinen (national \& international)

Religionen und Religionsgemeinschaften bzw. deren Vertreter*innen, Amtsträger*innen, Institutionen, religiöse Oberhäupter, etwa aus dem Christentum, Judentum oder anderen Religionen (außer Islam)

Islam im Speziellen (national \& international)

Muslimische Amtsträger, Repräsentanten, Oberhäupter, Organisationen oder Personen, die als Muslime dargestellt werden, auch "der Islam", "die Muslime" etc. Wird nur codiert, wenn Person/en in erster Linie als Muslime bzw. Vertreter*innen des muslimischen Glaubens adressiert werden. Liegt der Fokus auf dem Status als Migrant wird 92 annotiert.

Soziale/Medizinische Akteure (national \& international)

Rettungsdienste, Krankenhäuser, Ärzt*innen, Feuerwehren, Krankenkassen, karikative Einrichtungen, Sozialarbeiter*innen etc.

\section{Zivilgesellschaftliche Akteure (national \& international)}

NGOs, Vereine, Hilfsorganisationen (z.B. Amnesty International, Ärzte ohne Grenzen, Brot für die Welt, SOS; inkl. Sprecher*innen, Mitglieder), Bürgerinitiativen oder -bewegungen, Ehrenamt (inkl. einzelne ehrenamtlich Tätige), Sportvereine, -verbände und Sportler*innen 
Organisierte Kriminalität, z.B. Mafia, Piraterie oder Terror (z.B. ISIS, Al-Qaida, Daesh); wenn Vereinigung als „terroristisch“ o. ä. bezeichnet wird (auch wenn sie es in den Augen der Codierer*innen nicht ist, wird dieser Code trotzdem codiert)

70 Akteur aus Bildung, Forschung, Kultur (national \& international)

Personen und Organisationen aus Wissenschaft und Bildung (Universitäten, Institute, Schulen, Lehrer*innen, Professor*innen, Forscher*innen), auch Kindergärten, Kultureinrichtungen und-schaffende (Theater, Künstler*innen, Musiker*innen), Stiftungen, Fördervereine

80 Akteur aus Medienbereich (national \& international)

Journalist*innen und Medienorganisationen, Buch- und Presseverlage, öffentliche Anstalten, spezifische Medienangebote (z.B. einzelne Sendungen), auch Autor*in des Beitrags (!)

90 Personen in Deutschland (allgemein)

Angehörige der "Mehrheitsgesellschaft" (also nicht einer gesellschaftlichen Minderheit), z.B. deutsche Staatsbürger*innen ohne tatsächlichen oder mutmaßlichen Migrationshintergrund (z.B. "die Deutschen ", "Wir in Deutschland ", ",das Volk"); hier auch: Bewertungen anderen Kommentierenden codiert.

91 Rechtsextreme Personen/Gruppen in Deutschland

Spezifikation von 90: Personen in Deutschland, die offen fremdenfeindlich sind oder im Kontext des Beitrags so bezeichnet werden, z.B. "Nazis", "Rechtsextreme", "Rechte", auch wenn etwa AfD-Wähler*innen (nicht die Partei=10) oder PEGIDA-Anhänger*innen (nicht die Organisation=61) so bezeichnet werden.

$92 \quad$ Personen mit Migrationshintergrund in Deutschland

Personen in Deutschland mit tatsächlichem oder mutmaßlichem Migrationshintergrund (unabhängig von eigener Migrationserfahrung), deren Aufenthalt in Deutschland thematisiert bzw. hervorgehoben wird (z.B. „die Ausländer", „die Migranten“, „die Türken").

93 Geflüchtete und Asylbewerber im Speziellen

Spezifikation von 92: Personen, die aus ihrem Heimatland geflüchtet sind und z.B. in Deutschland oder einem anderen europäischen Land Asyl oder einen anderen Schutzstatus beantragt haben.

Personen aus dem Ausland

Alle Einzelpersonen oder Gruppen (ggf. auch sonstige Akteure), die nicht in 90 bis 93 eingeordnet werden können (z.B. Touristen, aber auch „Leute in Ägypten“) 


\section{Bewertungsdimensionen}

Wurde das Bewertungsobjekt identifiziert, wird durch das Anklicken der entsprechenden Checkboxen die Dimension(en) bestimmt, hinsichtlich derer die Person(en) oder Gruppen bewertet werden. Die Frage ist hier also: Was wird bewertet?

Markiert werden muss mindestens eine Dimension. Es können aber auch mehrere Dimensionen angegeben werden.

\section{unspezifisch/übergreifend}

Äußerung, die eine Person oder Gruppe allgemein - im Sinne von: unspezifisch - bewertet. (Kann nicht mit anderen Dimensionen kombiniert werden.)

- Beispiele für negative Bewertungen: "Scheißkanake“; „Du bist doch scheiße“; „Drei schwarze Neger, in meiner Heimat stehn drei schwarze Neger, die moecht ich nimmer sehn...".; "Herzlich Willkommen asozialer Abschaum und primitiver Bodensatz der moslemischen Dritten Welt!"

- Beispiele für ambivalente Bewertungen: „Man muss ihn nicht mögen, doch er hat in den wenigen Wochen mehr bewegt, als seine Vorgänger in 12 Jahren. "; "Dass Migranten auch aber sowas von rechts sein können-kann sich die urbane Mittelschicht nicht vorstellen."; "Die Deutschen sind gut und beknackt."

- Beispiele für positive Bewertungen: "Du bist super"; "Lasst endlich diese armen Flüchtlinge in Ruhe, die sind schon traumatisiert genug. Kein Mensch ist illegal und kein Mensch ist ein Vogel. Also sollte kein Mensch dazu gezwungen werden sich registrieren zu muessen"

\section{Gesundheit (mental/physisch)}

Mentale oder körperliche Gesundheit einer Person oder Gruppe bezieht.

- Beispiele für negative Bewertungen: „Ich schlage vor, dass die Nachzügler für jeweils ein paar Wochen in Merkels Elfenbeinturm untergebracht werden. So steckt sich zumindest niemand wichtiges mit nachgezügelten Krankheiten an."; "Vielleicht solltest du dir mal professionelle Hilfe suchen, anstatt hier das Forum zuzumüllen. "; „Ihr seid einfach nur degeneriert."; „Wer bringt diese Irren endlich in die Hoelle"; „Das gefällt der Hasswarze nicht!! Die Frau ist so voller Hass und Argwohn, dass er sich schon in Hautwucherungen bemerkbar macht! Die hat bestimmt überall Geschwüre und ist verwarzt, durch und durch!!!"

\section{Intelligenz}

Äußerung, die die Intelligenz oder geistige Leistung einer Person oder Gruppe bewertet.

- Beispiel für negative Bewertungen: „ihr seid so was von dumm..."; „Entschuldigen Sie bitte, aber wenn Sie Ihren letzten Satz tatsächlich ernst meinen, sind Sie ein Idiot!"

- Beispiele für ambivalente Bewertungen: „Mir sind 16\% AfDwähler auch zuviel, allerdings bleiben auch 84 $\%$ die nicht so dumm waren"

- Beispiel für positive Bewertungen: „Horst Seehofer ist der einzige mit Verstand und einem guten Charakter. Er hat mit allem recht was er sagt"; "Was für ein unglaublich intelligenter Mann!"; „Ein Mann mit Weitsicht und Rückgrat. Einer der letzten die wir haben. “; "Das Frau Waagenknecht sehr klug ist, weiß man schon länger. Das zeigen nicht zuletzt solche Aussagen"

\section{Aussehen / Hygiene}

Äußerung, die die körperliche Erscheinung einer Person oder Gruppe bewertet, $d$. h. ihr Aussehen, ihre (Körper-)Hygiene oder ihren Geruch.

- Beispiele für negative Bewertungen: „Und die Regierung laesst es erst geschehen und will jetzt mehr Fussfesseln verteilen. Vor lauter schlechter Laune habe ich bestimmt bald genau so eine Hackfresse wie die Illegalen. "; "Wir holen uns hier den hässlichsten Feind ins Land, den wir jemals gesehen haben"

- Beispiele für positive Bewertungen: "Wenn aber nun die optisch und geistig um Klassen überlegene Wagenknecht an der linken Seite Wähler abknabbert, ist der Merkel-Plan in Gefahr." 


\section{Charakter / Verhalten}

Bewertung des Verhaltens einer Person oder Gruppe gegenüber anderen oder bestimmter (damit ggf. verbundener) Charaktereigenschaften (z. B. Mut haben, lügen, anderen helfen, naiv sein). Darunter fallen Verhaltensweisen, die sich auf das Zusammenleben bzw. einen positiven oder problematischen Beitrag einer Person bzw. Gruppe zur Gesellschaft. Auch Bewertungen entsprechend zugeschriebener Motive fallen in diese Kategorie (wenn etwa gesagt wird, jemand wolle Deutschland ausnutzen oder abzocken).

- Beispiele für negative Bewertungen: „Dass hierher fahnenflüchtige junge muselmanische Männer kommen, die nicht in der Lage sind, gegen die Mörderbanden des IS zu kämpfen ist für mich Feigheit, das sind keine armseligen Flüchtlinge "; "Verlogene Ratten, das ganze Pack"; "Die Katze lässt das Mausen nicht und der Moslem nicht das Lügen. "; „Ich bin gegen eine Massenaufnahme teils von kriminellen Moslems, die nicht gewillt sind sich hier anzupassen"; "Es gibt jetzt schon massive Probleme mit dem Integrieren! Wobei die meisten sich hier aufführen wie die Axt im Wald und eine Forderungsmentalität an den Tag legen, wo man nur mit dem Kopf schütteln kann!", "Die Politiker bringen auch nur Scheiße zustande“; „Wenn man 5 oder mehr Kinder hat lebt mann sehr gut mehr als man jemals erarbeiten könnte. Viele können kaum lesen und schreiben und sind streng gläubige Moslems und werden hier über Generationen nicht arbeiten das ist die traurige Wahrheit. "; "Türken, die seit 50 Jahren hier wohnen, wählen eindeutig, es nichts angekommen in deren Köpfen. Integration, ein Wunschdenken."

- Beispiele für ambivalente Bewertungen: „Für mich kann man das ziemlich simpel einteilen: Es gibt diejenigen, die sich an die Regeln halten und diejenigen, die es nicht tun.

- Beispiele für positive Bewertungen: „Genauso gut hält mir aber fast jeder junge Flüchtling die Tür auf oder bietet sich an, meinen Einkauf zu tragen“; "Aber nicht vergessen, es gibt auch Menschen aus diesen Ländern, die hier wirklich arbeiten (insbes. in Jobs, die hier keiner mehr machen will, Ernte, Pflege, Schlachthof, Bau, usw.)"; "Wirklich schade. Da kommt mal jemand der sich integriert und nach wenigen Wochen besser Deutsch spricht als mancher Ausländer nach 3 Generationen in Deutschland und die schmeissen wir raus"; "Wer seine Grenzen aufgibt, gibt sein Land auf!"; "Ausser Afd und Pegida wehrt sich Keiner dagegen! ( $\rightarrow$ negative Bewertung der anderen = diffuses Bewertungsobjekt)“

Aber Achtung: Aussagen wie: „Wir sollten tolerant sein" ist nicht als eine (positive) Bewertung zu verstehen und wird hier nicht annotiert. (Bei diesem Beispiel handelt es sich um einer Forderung - nicht um eine Bewertung).

\section{Kultur}

Äußerung, die die Werte und Kultur einer Person/Gruppe bewertet, z. B. Aussagen über die Zivilisiertheit von Gruppen (z. B. durch die Zuschreibung einer 'primitiven' Kultur). Hierzu zählt auch die Aussage, eine Gruppe gehöre nicht zu Deutschland dazu.

- Beispiele für negative Bewertungen: „Ach, ihr meint die Glücksritter, Nomaden und Terroristen, die ihre Bunga Bunga Party hierher verlagern möchten?"; „Dieser Mensch mit seinem Glauben, seinen Ansichten, seiner Kultur, gehört nicht in die westlich zivilisierte Welt. Und in nicht allzu ferner Zeit wird das auch noch die letzte kulturelle Austauschverständnisgruppe der 68er-ff Generation kapieren "; "Islahme/Araber/Törken gehören NICHT zu Deutschland!"; ",Von daher ist die provozierte Massenzuwanderung von hauptsächlich jungen Männern, die zum großen Teil kognitiv, kulturell und mental nicht in eine aufgeklärte westliche Gesellschaft zu integrieren sind, nur eine Forcierung dieser Transformation."

- Beispiele für ambivalente Bewertungen: „Der Islam gehört nicht nach Deutschland als Religion aber die Menschen sollten die Weltoffenheit genießen das ist wichtig. deutsche tugende behalten aber Islam als Staatsreligion ablehnen"

- Beispiele für positive Bewertungen: "So ein Quatsch. Auch Migranten gehören dazu“

\section{Sexualität}

Äußerung, die sich auf sexuelles Verhalten einer Person/Gruppe bezieht, z. B. sexuelle Praktiken (z. B. Sex mit Tieren) und deren Häufigkeit, aber auch Prostitution. Achtung: Sexuelle Gewalt wird unter der Dimension 'Gesetzestreue' erfasst.

- Beispiele für negative Bewertungen: "Man könnte ja Sexroboter in Ziegenform bauen“; „Deutschland hat noch genügend Platz für alle wie die Karnickel rammelnden Menschen aus aller Herren Länder!", "du Hure!!!" 


\section{Gesetzestreue}

Äußerung, die sich auf die Gesetzestreue des Objekts bezieht. Eine negative Bewertung der Gesetzestreue liegt dabei vor, wenn jemandem ein kriminelles / illegales Verhalten zugeschrieben wird, gewalttätig zu sein oder ein Terrorist zu sein. Eine positive Bewertung der Gesetzestreue liegt dagegen vor, wenn jemandem ein kriminelles oder illegales Verhalten ab-oder ein gesetzestreues Verhalten zugesprochen wird.

- Beispiele für negative Bewertungen: „Sind das nicht alles nicht-asylberechtigte Trittbrettfahrer, also illegale?"; "Die BRD-Führungsclique ist krimineller als die kommunistische der DDR"; "Ich bin gegen eine Massenaufnahme von kriminellen Moslems, die nicht gewillt sind sich hier anzupassen"; "Ich meine er hat keinen Schuß, er verfolgt nur die deutsche und europäische Presse, die meist noch immer beschönigt. Hier auf das Gleis geschupst, angezündet, Treppe hinuntergetragen, usw. Das Leben wird von Tag zu Tag lebensgefährlicher.; „Dann halten Sie auch die Füße still, wenn es weiteren Anstieg an Kriminalität durch Asylanten gibt, Vergewaltigungen zunehmen"; "Ich will nicht ausprobieren wer von denen moderat oder vllt. gemäßigt ist, also sind für mich alle Terroristen"; „Junge Männer die vorher gemordet und vergewaltigt haben werden hier rundum versorgt."

- Beispiele für positive Bewertungen: „Sie besagen zum Beispiel, dass die Kriminalitätsrate von Ausländern nicht signifant höher ist als bei Einheimischen. Der Nordafrikaner neigt nicht mehr zu Vergewaltigung als der Nordsiegerländer. "; „Lasst endlich diese armen Flüchtlinge in Ruhe, die sind schon traumatisiert genug. Kein Mensch ist illegal und kein Mensch ist ein Vogel. Also sollte kein Mensch dazu gezwungen werden sich registrieren zu muessen."

\section{Wirkung auf Deutschland}

Äußerung, die sich auf die Wirkung des Objekts auf Deutschland bezieht. Gemeint sind damit Wirkungen auf die Kultur, Werte, das politische/gesellschaftliche System als solches oder ein einzelnes Teilsystem. Aussagen, das eine Person/Gruppe eine Gefahr für Deutschland sei, sind als negative Bewertung zu codieren.

- Beispiele für negative Bewertungen: „Wir holen uns hier den hässlichsten Feind ins Land, den wir jemals gesehen haben"; "Die Gutmenschenfrauen und Mütter werden wahrscheinlich vor ihrer Beerdigung noch ,Burka' tragen müssen, wenn das hier so weitergeht"; "Wenn endlich das Scharia Gesetz in Deutschland kommt, wird der Fall neu verhandelt und er kann auf eine frühzeitige Entlassung hoffen. Die Zeichen dafür stehen ja gut in Deutschland."; "Wenn wir uns von denen weiter auf der Nase rumtanzen lassen, werden wir alle sterben"; "Muslemische Terroristen werden unser aller Untergang sein"; „Wenn Merkel so weitermacht, werden wir alle sterben."

- Beispiele für ambivalente Bewertungen: "Schlecht ist wenn dabei nicht überprüft wird und radikale Leute aus Politik und Religion kommen. Die ganz und gar nicht vorhaben zu arbeiten, sondern nur um Anschläge zu machen oder sich Geld zusammenrauben."

- Beispiele für positive Bewertungen: „Wir wollen Menschen, die uns voranbringen und unsere Rente sichern"; „Vielen Dank Herr Elsässer. Ihre Arbeit ist unschätzbar wertvoll für unser Volk und kann nicht genügend gelobt werden. Auch sie haben sich ihren Platz in den Geschichtsbüchern redlich verdient." 


\section{Implikation}

Hier wird für jede Bewertung erfasst, ob ein bestimmter Umgang mit der Person/Gruppe nahegelegt wird. Falls dies nicht der Fall ist, wird „00 keine Implikation“ ausgewählt.

\section{Umgang (ohne Erwähnung von Gewalt)}

Äußerungen, die sich auf den (nicht-gewalttätigen) Umgang mit dem Objekt beziehen (was soll mit dem Objekt passieren / gemacht werden?)

- Beispiele für negative Bewertungen: „Das Pack muss raus“; „Daheim ists am schönsten, Jungs! Ihr schafft das!"; "verpisst euch doch einfach, keiner will euch hier haben :D“; "Wer betrügt fliegt"; "Wer sich auf Schleppern einläßt, Ausweise wegwirft, um in den Sozialstaat einzuwandern, darf gerne die Spur wechseln- zurück nachhause!!!"

- Beispiele für ambivalente Bewertungen: „Geht es vielen Politikern möglicherweise nicht eher darum, sogenannte integrierte Flüchtlinge hier zu behalten. Das geht ja in Ordnung, jedoch meines Erachtens nur unter der Bedingung, dass bestimmte Mangelberufe ausgeübt werden."; "Wer sich hier ohne Arbeit nicht finanzieren kann, der muss wieder gehen!"

- Beispiele für positive Bewertungen: „Handelt es sich um einen Flüchtling, der eine Tätigkeit gelernt hat, welche wir langfristig dringend brauchen werden, der die deutsche Sprache spricht, der ein gesichertes und ausreichendes Einkommen und der keine Straftaten oder Ordnungswidrigkeiten begangen hat, dann soll der doch bitte bleiben"

Hier wird codiert, ob der Kommentar eine Äußerung enthält, die explizit oder implizit zu tätlicher Gewalt gegen die Person/Gruppe aufruft bzw. explizit oder implizit mit einer Gewaltanwendung droht oder diese gutheißt

- Beispiele für negative Bewertungen: „Keine Frage das dieser in die Sicherungsverwahrung muss. Und hoffentlich hat er nette Mitgefangene die ihn in dieser Zeit ordentlich rannehmen. "; "Glauben Sie mir, das war gewollt. Und auch, wenn es nicht gewollt war, gehören die verantwortlichen Personen mit Mistgabeln und Fackeln davon gejagt. Denn was besseres gestattet unser GG uns nicht. "; „Unsere Flugzeugträger erlauben es uns euch in die Steinzeit zurückzubomben!"; „Und soll ich mal ganz ehrlich was sagen: es wäre mir auch sch...egal, wenn er menschenrechtswidrig behandelt würde."

Hier wird codiert, ob der Kommentar eine Äußerung enthält, die explizit oder implizit mit der Tötung einer Person oder der Auslöschung einer Gruppe droht bzw. andere explizit oder implizit dazu aufruft oder die (Selbst-)Tötung einer Person oder die Auslöschung einer Gruppe explizit oder implizit gutheißt

- Beispiele für negative Bewertungen: „Hoffentlich kommt der Heilige Krieg nach Europa. Wir Russen und Osteuropäer werden euch Abschlachten. "; "Aber das Pack sind Deserteure, die standrechtlich zu erschießen sind. "; "Warum erst verhaftet, gleich unter die Dusche. Das kostet den Steuerzahler am wenigsten"; "Gebt der Schlampe einen Baum!"; "Adi hätte es gemacht wie damals mit dem Judenpack und hätte die Kammern und die Öfen wieder an gemacht is doch genug zum Gasen und verbrennen hier her gekommen. "; "Wäre der Terrorist ein Bankster, hätte er das Bedürfnis zufällig vom Hochhaus zu springen!!!" 


\section{Gruppenbezüge (Verallgemeinerung)}

Hier wird festgehalten, ob und an welchen vermeintlichen oder tatsächlichen Eigenschaften des Bewertungsobjekts die Bewertung festgemacht (und dadurch verallgemeinert) wird, d. h. sofern keine Verallgemeinerung stattfindet, wird keines der folgenden Attribute markiert. Es können auch mehrere Verallgemeinerungen markiert werden, z. B. wenn ein verallgemeinerndes Urteil über Männer aus Syrien gefällt wird.

\section{Ethnie}

Ethnische Zugehörigkeit, Nationalität, Herkunft, Abstammung, Rechtsstatus (bzgl. Einwanderung)

- Beispiele für negative Bewertungen: „Was bringt uns eine Person aus Ghana, Eritrea oder Tunesien die angeblich hier ein Jobangebot hat, dann doch arbeitslos wird und einen Asylantrag wegen (angeblicher) Homosexualität stellt?"; "Asylpack"

- Beispiele für positive Bewertungen: "Meistens sind Iraner sehr gut integrierbar"; "Gerade wir alten Migranten, die eine Menge dazu beigetragen haben, dass dieses Land vorangeht, sollten mit gutem Beispiel vorangehen "; "Genauso ist es. Die Rumänen in der Gastronomie sind fleißige, ehrliche anständige Leute."

\section{Religion}

- Beispiele für negative Bewertungen: „Der Islam sorgt, wie wir es alle täglich sehen, weltweit für Krieg, Gewalt und Terror. Kein einziger islamischer Staat mit demokratisch gewählter Regierung, ohne Terror."

\section{Geschlecht}

- Beispiele für negative Bewertungen: „Es gibt kein anderes Land außer vielleicht Schweden, das die eigenen Bürger derart verrät und der Gewalt ausländischer Männer preisgibt."

\section{Beruf}

- Beispiele für negative Bewertungen: „Sie werden es kaum abstreiten können, dass der deutsche Journalismus seit geraumer Zeit weder objektiv noch investigativ berichtet" 


\section{Sprachliche Formen}

Mithilfe der folgenden Attribute wird die Form der Bewertung näher charakterisiert. (Wie wird die Bewertung formuliert oder ausgedrückt?). Wieder gilt: Wenn keine der folgenden Formen in einer Bewertung auftritt, wird auch kein Attribut markiert. Umgekehrt können auch mehrere Attribute für eine Bewertung markiert werden.

\section{Ironie/Sarkasmus}

Hier wird als Attribut erfasst, ob die Bewertung durch eine ironisch gemeinte Aussage ausgedrückt wird. Erfasst werden sollen damit Aussagen, die nicht so gemeint sind wie sie formuliert sind, sondern das Gegenteil ausdrücken sollen. Ironie kann z. B. an Übertreibungen oder an "Anführungszeichen“ erkannt werden.

- Beispiele für ironisch gemeinte Bewertungen: „„,Pflegekraft Abeba: kompetent, motiviert'- so wie alle 975 Milliarden Afrikaner. Schade, dass sie nicht aller hier sein können. Vielleicht nächstes Jahr. “; „Aber wie bereits erwähnt: Die Afrikaner werden es richten. Die sind total fleißig und daran interessiert, solche Arbeiten zu erledigen. Unsere Senioren freuen sich bestimmt auch darauf."; "Das kann doch gar nicht sein, denn die Gäste sind doch alle so nett, fröhlich und gutgelaunt", "von den hoch spezialisierten Goldstücken beglückt werden..."

\section{Beschimpfung}

Hier wird als Attribut erfasst, ob die Bewertung eine (vulgäre) Beschimpfung enthält. Gemeint sind Schimpfworte und abwertende Personen- bzw. Gruppenbezeichnungen.

- Als Schimpfwörter gelten Personenbezeichnungen, die der bezeichneten Person eine bestimmte, negativ-konnotierte Eigenschaft oder Verhaltensweise zuschreiben (z. B. "Schlampe", "Hure", "Schwuchtel", „Hurensohn“, ,Bastard", "Schwein", „Arschloch", ,Idiot", ,Ziegenficker", "Schlitzauge" , "Untermensch") oder die konventionell einen negativen Verwendungszusammenhang aufweisen (z. B. "Kanake", „Zigeuner", "Gesocks").

- Der Gebrauch einer abwertenden Wortbildung zur Bezeichnung der Person/Gruppe (z. B. Präfix "Scheiß-" oder „Drecks-“ oder Suffix „-pack") gilt als Beschimpfung.

\section{Metaphern und Vergleiche}

Hier wird als Attribut erfasst, ob im Rahmen der Bewertung auf Metaphern, Sprachbilder oder Vergleiche (z.B. Tiervergleiche) zurückgegriffen wird, durch die die bewerteten Personen oder Gruppen z.B. als Gefahr dargestellt und ggf. entmenschlicht werden. Beispiele sind hier Metaphern und Vergleiche aus den folgenden Bereichen:

- Wasserlauf, Naturgewalten, Naturkatastrophen, z.B. "Flüchtlingswelle“, "-strom"

- Krieg und Invasion, z.B. "Invasoren ", "Armee“, "Angriff" ",Front"

- Medizin, Hygiene, Krankheiten, z.B. "Schädlinge", "Parasiten" ",Fremdkörper"

- Tiere, z.B. "Ratten", „Kakerlaken", "Horden ", "Schwärme", ,Instinkte“, „Angela Ferkel"

- Pflanzen, z.B. "keimende Saat", "Spreu vom Weizen", "Entwurzelung", ",Wildwuchs"

- Schifffahrt und Warenverkehr, z.B. "Fracht", "geschmuggelt", "Reißleine ziehen"

- Tourismus, z.B. „Asyltourismus"

\section{Formale Verfremdung}

Hier wird als Attribut erfasst, ob ein Kommentar Begriffe mithilfe von Sonder- oder Satzzeichen verfremdet, z.B. „...du VRSSCH..." oder „...du A_R_S_C_H..."; „sch*iß"; "Die Juden die grö SS ten Feinde der Muslims" 


\section{Abwertende Umschreibungen}

Hier wird als Attribut erfasst, ob die Bewertung ein (abwertendes) Wortspiel / eine (abwertende) Wortschöpfung enthält. Gemeint sind Ausdrücke, Kombinationen, Abwandlungen oder Bindestrichwörter, durch die einer Person, Gruppe, Nationalität oder Religion negative Eigenschaften oder Verhaltensweisen zugeschrieben werden, z.B. "ISISlam" , "Rapefugee“, "Angela Ferkel", "Surensöhne“, "Messer-Migranten", „Goldstücke“, "Gutmenschen", "Flüchtlingsschauspieler", „Fluchtsimulanten"; auch distanzierendes In-Anführungsstriche-Setzen (z.B. "diese sog. 'Flüchtlinge'" zählt hierzu), ",SurenSöhne aus Schwarz-Afrika"; "Zeilengeldnutten der politischen Propagandafront"; ",Merkill"; "NaziAltparteien-Kartelle"

\section{Entmenschlichung}

Wird als Attribut erfasst, wenn die Bewertung sprachlich (auch) durch eine Gleichstellung mit als eklig oder negativ konnotierten Tieren, anderen nicht-menschlichen Wesen (wie z. B. Teufel, Vampire, Kannibalen) oder Objekten ausgedrückt wird, etwa durch die Verwendung von Tiermetaphern oder-vergleichen (s.o.), die Charakterisierung als wild/animalisch oder die Bezeichnung als „Abschaum" oder „Pack". Durch die Bewertung wird dem Objekt die Daseins-Berechtigung abgesprochen.

- Beispiele: "Verlogene Ratten, das ganze Pack“; „Deutschland hat noch genügend Platz für alle wie die Karnickel rammelnden Menschen aus aller Herren Länder!"; "Man könnte fast meinen, dass man den Tieren damit Unrecht tut, wenn man dieses Individuum mit ihnen auf eine Stufe stellt. "; "Allahu Ackbah Orks"; "Die Volksdeutschen sollen sich gefälligst widerstandslos abschlachten lassen von Merkels 666er-Orks"; „Die Linke und die grünen Tuschlechten quieken wie die Schweine angesichts ihrer Niederlagen. Verzeihung, ich nehme den unpassenden Vergleich zurück, Schweine sind liebenswerte Tierchen..."; „Sozialschmarotzer" 